\title{
Laparoscopic splenopexy for wandering spleen with absorbable mesh fixation
}

\author{
D Nel, ${ }^{1}$ iD C Kloppers, ${ }^{1}$ iD E Panieri ${ }^{1}$ iD \\ Division of General Surgery, Groote Schuur Hospital, University of Cape Town, South Africa
}

Corresponding author, email: danielnel87@gmail.com

\begin{abstract}
Summary
Wandering spleen is a rare condition characterised by lack of fixation of the spleen to its usual position in the left upper quadrant. Laparoscopic splenopexy has become the standard of care. Although a number of methods have been described, the use of an absorbable mesh to secure the spleen to the left upper quadrant has been shown to be safe, effective and quick to perform. We present a case of a 32-year-old female with this condition and describe our surgical technique for fixation of the spleen using an absorbable mesh.

Video available online: http://sajs.redbricklibrary.com/index.php/sajs/article/view/3531
\end{abstract}

\section{Case description}

\section{Introduction}

Wandering spleen (WS) is a rare condition characterised by a lack of fixation to the left upper quadrant. This allows the spleen to be mobile within the peritoneal cavity, often having a longer vascular pedicle, putting it at risk for torsion and infarction. ${ }^{1,2}$ WS is seen most commonly in children and women of childbearing age. Clinically, it may present as an abdominal mass or as acute abdominal pain when torsion occurs. ${ }^{1,2}$ Episodes of torsion and spontaneous detorsion may occasionally occur and give rise to a chronic history of intermittent abdominal pain. ${ }^{2}$ The diagnosis is rarely suspected clinically and is usually based on characteristic findings on abdominal ultrasound or CT, with the spleen noted to be absent from its normal position and a mass seen elsewhere in the abdomen. In addition, a "whirl" appearance of the splenic pedicle on CT suggests torsion in acute cases. ${ }^{3}$

The true natural history of asymptomatic patients with WS is unknown, but in those in whom the diagnosis is confirmed, conservative management results in complication rates as high as $65 \% .{ }^{1}$ Thus, the management is surgical, with the timing of surgery depending on whether the patient has acute torsion with splenic ischaemia. ${ }^{2}$ Although splenectomy is historically the most widely performed procedure, and is still used for cases where torsion has resulted in infarction, splenic preservation with splenopexy has gained popularity, particularly in paediatric patients, avoiding the risks of overwhelming post-splenectomy infection. ${ }^{1,45}$ Early diagnosis in cases of acute torsion is key to allow for splenic preservation.

\section{Case presentation}

Our patient was a 32-year-old mother of two small children. She presented with recurrent episodes of severe central abdominal pain, radiating to the left lumbar region over a three-year period. Pain was often experienced post- prandially, and was occasionally associated with nausea. At the time of the index admission, she complained of very severe discomfort, but clinical examination failed to reveal any striking findings. Particularly, she did not have features of peritonism or palpable mass. A CT scan was ordered, which showed a mildly enlarged spleen located in the left flank region, with obvious torsion of the hilum and tail of pancreas. The patient's pain resolved spontaneously within 12 hours, and she was booked for semi-urgent surgery.

The patient was placed in the supine position with a 15 degree lateral tilt. A $10 \mathrm{~mm}$ umbilical port was placed under vision at the umbilicus, with an additional two $5 \mathrm{~mm}$ working ports, one in the epigastrium and the other in the right iliac fossa.

The spleen was noted to be viable, normal in size and lying in the left upper quadrant (LUQ), but easily movable to the central abdomen. It was lying anteriorly to a mobile loop of descending colon, which appeared dilated as a result of this relation. The colon was also noted to be poorly fixated to the retroperitoneum, but otherwise macroscopically normal. A nasogastric tube was inserted intraoperatively to deflate the stomach and was removed prior to extubation.

A 30 x $30 \mathrm{~cm}$ Polyglactin/"Vicryl" mesh was reduced in size to match the volume of the spleen, and was inserted through the umbilical port. The mesh was placed in the LUQ and the spleen placed on it. The mesh was then folded over, and wrapped anteriorly around the spleen. The edges of the mesh were secured together with clips and $3 / 0$ vicryl suture to manufacture a secure holding structure, making sure not to compress the hilar vessels. Finally, the lateral aspect of the mesh was secured to the abdominal wall in the LUQ with $2 / 0$ barbed "V-Loc" sutures to achieve a 3-point fixation. The colon was placed anterior to the tail of the spleen, such that fibrosis induced by the mesh bag, fixed to the diaphragm, would prevent the colon from migrating behind the spleen and potentially obstructing in future. The duration of surgery was 98 minutes. 
The patient had an uneventful postoperative course and was discharged the day after the surgery. Over the subsequent weeks, she complained of persistent discomfort in the LUQ, and mild nausea. This was attributed to a presumed inflammatory reaction triggered by the intraperitoneal vicryl mesh, and was easily managed with simple analgesics for the first ten days. Her clinical condition gradually improved, and she was asymptomatic and fully active at follow-up after four weeks. The patient was contacted telephonically at 12 months after surgery and remained well with no repeat episodes of severe abdominal pain suggesting recurrent splenic volvulus.

\section{Discussion}

As awareness of the importance of the spleen's immune function has grown, splenectomy has become less popular and is now reserved for cases of gross splenomegaly, hypersplenism or massive splenic infarction. Where partial infarction occurs, this can be managed with partial splenectomy, followed by splenopexy. ${ }^{6}$

A number of operative approaches for splenopexy have been described. Open methods have included fixing the splenic hilum to the splenic bed, placing the spleen in a retroperitoneal pocket in the LUQ, using adjacent organs to create an intraperitoneal pocket, and direct suturing of the splenic capsule to the diaphragm/abdominal wall. Wrapping the spleen in an absorbable mesh bag, followed by fixation in the LUQ was first described in 1992 by Schmidt et al., who also showed that the spleen remained anchored in this position at 4-year follow-up.?

Laparoscopic approaches, with the concomitant advantages of minimally invasive surgery, have become increasingly popular. ${ }^{1,2,4,8,9}$ Although laparoscopic techniques for creation of an intra- or extra-peritoneal pocket in the LUQ for the spleen have been described, the use of absorbable mesh to wrap around the spleen and secure it has become the most popular technique. This method appears to be safe, effective and quick to perform. ${ }^{5,6,8}$ Even for patients with splenomegaly, where creation of a pocket with natural tissue may be challenging, a sandwich technique using two pieces of mesh can still be performed with relative ease. ${ }^{10}$

In conclusion, we report a case of a 32-year-old female with wandering spleen managed successfully with laparoscopic splenopexy using an absorbable mesh.

\section{Conflict of interest}

The authors declare no conflict of interest.

\section{Funding source}

None.

\section{ORCID}

D Nel (iD https://orcid.org/0000-0002-3265-1049

C Kloppers iD https://orcid.org/0000-0003-2438-6879

E Panieri (iD https://orcid.org/0000-0002-7907-8497

\section{REFERENCES}

1. Soleimani M, Mehrabi A, Kashfi A, et al. Surgical treatment of patients with wandering spleen: report of six cases with a review of the literature. Surg Today. 2007;37(3):261-9. PMID: 17342372. https://doi.org/10.1007/s00595-006-3389-0.

2. Montenovo MI, Ahad S, Oelschlager BK. Laparoscopic splenopexy for wandering spleen: case report and review of the literature. Surg Laparosc Endosc Percutan Tech. 2010;20(5):e182-4. PMID: 20975498. https://doi.org/10.1097/ SLE.0b013e3181f69ce2.

3. Ely $\mathrm{AB}$, Zissin R, Copel L, et al. The wandering spleen: CT findings and possible pitfalls in diagnosis. Clin Radiol. 2006;61(11):954-8. PMID: 17018308. https://doi. org/10.1016/j.crad.2006.06.007.

4. Cavazos S, Ratzer ER, Cavazos ME. Laparoscopic management of the wandering spleen. Surg Laparosc Endosc Percutan Tech. 2004;14(4):227-9. PMID: 15345161. https:// doi.org/10.1089/lap.2004.14.227.

5. Allen KB, Andrews G. Pediatric wandering spleen - the case for splenopexy: review of 35 reported cases in the literature. J Pediatr Surg. 1989;24(5):432-5. PMID: 2661792. https://doi. org/10.1016/s0022-3468(89)80395-1.

6. Fonseca AZ, Ribeiro Jr M, Contrucci O. Torsion of a wandering spleen treated with partial splenectomy and splenopexy. J Emerg Med. 2013;44(1):e33-6. PMID: 22381612. https://doi. org/10.1016/j.jemermed.2011.06.146.

7. Schmidt SP, Andrews HG, White JJ. The splenic snood: an improved approach for the management of the wandering spleen. J Pediatr Surg. 1992;27(8):1043-4. PMID: 1403532. https://doi.org/10.1016/0022-3468(92)90555-1.

8. Palanivelu C, Rangarajan M, Senthilkumar R, et al. Laparoscopic mesh splenopexy (sandwich technique) for wandering spleen. JSLS. 2007;11(2):246. PMID: 17761090.

9. Hedeshian MH, Hirsh MP, Danielson PD. Laparoscopic splenopexy of a pediatric wandering spleen by creation of a retroperitoneal pocket. J Laparoendosc Adv Surg Tech A. 2005;15(6):670-2. PMID: 16366881. https://doi.org/10.1089/ lap.2005.15.670.

10. Nomura H, Haji S, Kuroda D, et al. Laparoscopic splenopexy for adult wandering spleen: sandwich method with two sheets of absorbable knitted mesh. Surg Laparosc Endosc Percutan Tech. 2000;10(5):332-4. PMID: 11083221. 